\title{
Meta
}

Journal des traducteurs

Translators' Journal

\section{Blick in die Black Box: Kreative Momente im Übersetzungsprozess: eine experimentelle Studie mit Translog}

\section{Tanja Heiden}

Volume 50, numéro 2, avril 2005

Processus et cheminements en traduction et interprétation Processes and Pathways in Translation and Interpretation

URI : https://id.erudit.org/iderudit/010993ar

DOI : https://doi.org/10.7202/010993ar

Aller au sommaire du numéro

Éditeur(s)

Les Presses de l'Université de Montréal

ISSN

0026-0452 (imprimé)

1492-1421 (numérique)

Découvrir la revue

Citer cet article

Heiden, T. (2005). Blick in die Black Box: Kreative Momente im Übersetzungsprozess: eine experimentelle Studie mit Translog. Meta, 50(2), 448-472. https://doi.org/10.7202/010993ar
Résumé de l'article

Dans cet article, le processus de traduction est pris en considération sous l'angle des sciences cognitives. L'accent sera mis sur un élément essentiel de l'opération traduisante et qui pour cette raison mérite être étudié de manière plus détaillée et approfondie : il s'agit de la créativité en tant qu'activité servant à résoudre des problèmes de traduction. Mais qu'est-ce que la créativité en traduction exactement? Quels sont les éléments qui la composent ? Quand, à savoir à quels moments durant le processus de traduction, et comment, en d'autres termes, par quels facteurs, ce phénomène est-il produit et activé ? Voici seulement quelques questions, auxquelles nous allons tenter de répondre dans le cadre de notre étude ainsi que dans cette contribution. 


\title{
Blick in die Black Box: Kreative Momente im Übersetzungsprozess: eine experimentelle Studie mit Translog
}

\author{
TANJA HEIDEN \\ Universität Genf, Genf, Schweiz \\ Tanya.Heiden@eti.unige.ch
}

\begin{abstract}
RÉSUMÉ
Dans cet article, le processus de traduction est pris en considération sous l'angle des sciences cognitives. L'accent sera mis sur un élément essentiel de l'opération traduisante et qui pour cette raison mérite être étudié de manière plus détaillée et approfondie: il s'agit de la créativité en tant qu'activité servant à résoudre des problèmes de traduction. Mais qu'est-ce que la créativité en traduction exactement? Quels sont les éléments qui la composent? Quand, à savoir à quels moments durant le processus de traduction, et comment, en d'autres termes, par quels facteurs, ce phénomène est-il produit et activé? Voici seulement quelques questions, auxquelles nous allons tenter de répondre dans le cadre de notre étude ainsi que dans cette contribution.
\end{abstract}

\begin{abstract}
The following article considers the translation process from the point of view of cognitive language processing. The emphasis here is on a single notion, creativity, which appears very often in the field of translation and will thus be studied in more detail. However, what exactly is creativity? When, i.e., at what stages of the translation process, does it occur? And how is it activated, i.e., which are the factors that trigger creativity? These are some of the questions that we will try to answer in the course of our research and in this article.
\end{abstract}

\section{MOT-CLÉS/KEYWORDS}

Übersetzungsprozess, Kreativität, Definitionen von Kreativität, Vorkommen kreativer Momente im Übersetzungsprozess, Problemlösen

\section{EINLEITUNG}

Vorweg gilt es dem Leser vielleicht zu präzisieren, auf welche Grundannahmen sich dieser Beitrag stützt: An Anlehnung an die Auffassung der beiden Gründerväter ${ }^{1}$ der Kreativitätsforschung, gehen auch wir davon aus, dass kreatives Denken in unserem Gehirn als Denkform angelegt ist und einem jeden Menschen ein kreatives Potenzial innewohnt, das entsprechend förderbar ist. Weiters erwähnt seien an dieser Stelle auch gleich die besonderen Schwierigkeiten, die es bei einer Untersuchung des Kreativitätsaspekts zu beachten gilt: Kreativität ist, wie wir alle bestens wissen, nicht leicht messbar ${ }^{2}$ und bewertbar und generell nur in einem ganz begrenzten Rahmen fassbar und nachvollziehbar. Schliesslich kann Kreativität noch aus Sichtweise verschiedener Disziplinen und Perspektiven betrachtet werden. Interdisziplinär sowie empirisch behandelt wurde dieses Thema $u$. W. nach jedoch erst seit den späten neunziger Jahren und dies im deutschsprachigen Raum lediglich bei Paul Kussmaul 
anhand von Protokollen des Lauten Denkens ${ }^{3}$. Paul Kussmauls folgend versuchen auch wir uns dem Thema im Spannungsfeld der Translationswissenschaften sowie der Kognitiven Wissenschaften zu nähern, jedoch nicht ausschliesslich aus Sichtweise der Kreativitätsforschung sondern auch aus dem Blickwinkel der Problemlösungsforschung, wobei immer wieder versucht werden soll, den kognitions-psychologischen mit dem translatorischen Blickwinkel miteinander zu korrelieren und die wissenschaftlichen belegten Erkenntnisse der beiden Wissenschaften auf den Übersetzungsprozess zu übertragen.

\section{KONZEPT DESKREATIVEN PROBLEMLÖSENS}

\subsection{Problemlösen vs. Kreatives Problemlösen}

\section{Was bedeutet Problemlösen?}

Zur Definition eines Problems und dessen Natur ist zu sagen, dass „ein Problem (...) dadurch gekennzeichnet ist, dass

a. ein Ausgangszustand vorliegt, welcher

b. in einen erwünschten Ziel- oder Endzustand überführt werden soll, jedoch

c. Schwierigkeiten, Hindernisse oder Barrieren existieren, die einer direkten und momentanen Überführung des Ausgangszustandes in den Endzustand im Wege stehen. ${ }^{4}$

\section{Was bedeutet kreatives Problemlösen?}

In Anlehnung an Joachim Funke $(2003)^{5}$ bedeutet kreatives Problemlösen das „Hervorbringen eines neuen, individuell oder gesellschaftlich nützlichen Produkts, das nicht durch Anwendung von Routineverfahren zu erzeugen ist“.

Bei einer näheren Betrachtung des Kreativitätsbegriffs im Zusammenhang mit Problemlösen wird ersichtlich, dass der Schwerpunkt verschiedenartig gelagert werden kann. Eine Untersuchung kann somit sein:

- subjektzentriert: auf die kreative Person gerichtet;

- prozessorientiert: auf den kreativen Prozess gerichtet oder

- produktzentriert: auf das kreative Produkt gerichtet.

Es stellt sich vorab die Frage, welche Art der Problemlösung als kreativ zu bezeichnen ist. Mit anderen Worten: Was unterscheidet allgemeines Problemlösen von kreativem Problemlösen? Laut Hussy (2004) ist die Art der Neuverknüpfung beim kreativen Prozess selten (wobei dies unserer Ansicht nach kein wirklich objektivierbarer und messbarer Faktor ist), bezieht sie sich auf ein umfangreiches bereichsspezifisches Faktenwissen und folgt keinem gängigen Lösungsweg.

\section{Kreativität im Übersetzungsprozess als Untersuchungsgegenstand:}

Während unserer Überlegungen zum Kreativitätsbegriff als Problemlösungsaktivität im Übersetzungsprozess sind generell folgende Fragen aufgetaucht: Welche Möglichkeiten der allgemeinen Lösungsfindung gibt es beim Übersetzen? Welche Möglichkeiten der kreativen Lösungsfindung gibt es? Oder, wie kann ein Übersetzer eben solche Übersetzungsprobleme meistern, die nicht nach analytischen, formal-logischen, rationalen und konventionellen Denkprozessen ablaufen und auch nach erfolgter 
Recherchierarbeit in Bezug auf die Terminologie oder den Gegenstand nicht lösbar sind? Ist zur Lösungsfindung dann nicht etwa Kreativität angebracht oder zumindest gefragt - als Art der Entscheidungsfindung in einer komplexen Übersetzungsproblemssituation? Was genau unterscheidet kreative Problemlösungsprozesse beim Übersetzen von allgemeinen Problemlösungsprozessen? Bringen kreative Problemlösungsprozesse jedes Mal auch kreative Übersetzungsleistungen mit hervor? Welche sind die psychologischen Faktoren oder Ursachen, die sie bedingen? Wie können kreative Problemlösungsstrategien aussehen, die zu kreativen Übersetzungsvorschlägen führen? Sind diese retroaktiv überhaupt nachweisbar und analysierbar? Sind diese dank psycholinguistischer Methoden, wie der Anfertigung und Analyse von Protokollen Lauten Denkens oder neuer Softwareprodukte wie beispielsweise Translog vielleicht sogar in vivo beobachtbar und beschreibbar? Wenn man den Übersetzungsprozess weiters, anlehnend an Krings in drei Phasen (eine Einführungs-, Transferund Revisionsphase) zu unterteilen versucht, drängt sich die Frage auf, wann es im gesamten Übersetzungsprozess zu solchen kreativen Momenten kommt, die in der Folge zu einer kreativen Übersetzungslösung führen? Gibt es bestenfalls auch Techniken zur Stimulierung, Aktivierung oder Förderung von Kreativität, die im Übersetzungsunterricht auch erfolgreich eingesetzt werden könnten? All dies sind Fragen, auf die wir im Rahmen unserer Untersuchung ${ }^{6}$ Antworten zu finden versuchen.

\subsection{Annäherungsweisen an den Kreativitätsbegriff}

\subsubsection{Produktorientierte Sichtweise nach Barron (1955) \& Stein (1974)}

Dem Definitionsversuch von Barron (1955) und Stein (1974) zufolge, sollte die Lösungsfindung sowohl ungewöhnlich (vgl. Hussy, demnach der Prozess keinem gängigen Lösungsweg folgen soll) als auch nützlich sein.

\subsubsection{Personenzentrierte Sichtweise und Analyse nach Guilford}

Nach dem ACL-Verfahren (Creative Personality Score for Adjektive Checking List) fand Gough (1979) in der Weiterentwicklung von Guilfords ${ }^{7}$ Eigenschaftsmuster für kreativ denkende Personen, dass diese „fähig, interessiert, zuversichtlich, unkonventionnell, vielfältig, intelligent, selbstsicher usw. “ und für nicht-kreativ denkende Personen, dass diese „vorsichtig, konservativ, unzufrieden, ernst usw.“ seien.

Eine kreative Person zeichne sich - so wird es in der einschlägigen Literatur immer wieder betont - ausserdem vor allem durch folgende Eigenschaften aus: Intellektuelle Fähigkeiten, Wissen, Persönlichkeit und Motivation. $\mathrm{Zu}$ den intellektuellen Fähigkeiten zählen - wenn wir an John Deweys Ausspruch „A problem well put is half solved" denken - zunächst das Entdecken und Definieren eines Problems, die Repräsentation eines Problems, die durch visuelle Vorstellungen erfolgen kann, die Auswahl einer Strategie durch heuristische Suche, divergentes Denken (im Unterschied zu konvergentem Denken) sowie die endgültige Bewertung. Schliesslich ist aber Motivation die eigentliche Treibkraft, die kognitive Ressourcen überhaupt erst zu kreativen Ideen und Handlungen bringt.

In diesem Beitrag soll jedoch in erster Linie ein kognitionspsychologischer Ansatz verfolgt werden, der, wie bereits angedeutet, nicht vordergründig vom kreativen Pro- 
dukt oder von der kreativen Person, sondern vor allem wie im folgenden in groben Zügen skizziert von Kreativität als Prozess ausgeht.

\subsubsection{Prozessorientierte Sichtweise nach Newell, Shaw \& Simon (1962)}

Newell, Shaw und Simon ${ }^{8}$ sehen als grundlegendste Unterscheidungsmerkmale zwischen kreativem Problemlösen und allgemeinem Problemlösen die Zurückweisung bisher akzeptierter Ideen, motivationales Engagement sowie die Präzisierung eines vagen Problems beim Prozess der Lösungsfindung (vgl. die auch heute noch vertretene moderne Auffassung von Kreativität als Prozess nach Hussy 1992, 1996, 2004). Der erste Punkt kann dabei wahrscheinlich als das wichtigste Unterscheidungsmerkmal gelten: Beim Problemlösen ist per definitionem nur die Neukombination von Informationen zur Lösungsfindung gefordert, nicht aber die Zurückweisung von geläufigen Ideen.

In Anlehnung an sein Beispiel der Erfindung der Solarzelle zur Energieversorgung eines Raumschiffs zeigt sich die Zurückweisung bisher akzeptierter Ideen in der Abkehr von gängiger Denkweise (Strom aus dem Generator) und dadurch der Eröffnung neuer, noch unbegangener Wege. Zu Motivation und Persistenz ist vielleicht zu sagen, dass diese bei kreativen Prozessen ebenso gefordert ist wie bei allgemeinen Denkvorgängen, womit es schwer zu begründen wird, weshalb diese als Unterscheidungsmerkmal benutzt werden sollen bzw. könnten. Dasselbe gilt, unserer Ansicht nach, für das letzte Element zur Unterscheidung, das unter Umständen zwar zur Klassifikation von Problemarten herangezogen werden kann, jedoch nicht zur klaren Abgrenzung von allgemeinem Problemlösen.

\subsection{Aspekte des Kreativen Problemlösens nach Hussy}

\subsubsection{Kreatives Problemlösen und Fixierungen}

\section{Aufbau und Überwindung}

„Mit dem Zurückweisen gängiger Lösungswege rücken (...) Fixierungsphänomene, die wir als die verringerte Verfügbarkeit von Fakten- und Veränderungswissen zu verstehen haben, in den Mittelpunkt der Betrachtung" (Hussy 2004).

Es stellte sich hierbei die Frage, ob sich Personen mit hoch ausgeprägter Fähigkeit zum kreativen Problemlösen etwa leichter von solchen Fixierungen lösen können und deshalb zu ungewöhnlicheren Lösungsansätzen finden als andere? Man gab einer Person z. B. eine Kerze, ein Streichholzbriefchen und eine Schachtel Reissnägel und forderte sie auf, die Kerze so an einer hölzernen Tür zu befestigen, dass man Licht zum Lesen hat. Die Kerze musste richtig brennen ${ }^{9}$ Diese Problemstellung wurde dann in der Folge leicht modifiziert. Die Personen wurden zusätzlich instruiert, die Kerze nicht zu beschädigen und die Türe nicht mit Wachs zu verschmieren. Ausserdem waren die Reissnägel nicht lang genug, um eine direkte Befestigung der Kerze zu ermöglichen. Diese Abwandlungen erzwangen dann die Plattformlösung und ermöglichten die Überwindung der Merkmalsfixierung.

Hussy (1992) stellte sich als nächstes die Frage, ob Personen mit hoher Fähigkeit zum kreativen Problemlösen die Plattformlösung schneller finden würden als Personen mit diesbezüglich weniger ausgeprägten Fähigkeiten. Die Vpn wurden dafür 
nach dem Verbalen Kreativitätstest von Schoppe (1975) in zwei Gruppen eingeteilt: Nach hoher bzw. niedriger Fähigkeit zum kreativen Problemlösen. Die Ergebnisse bestätigten seine Erwartungen. In der Standardbedingung (modifizierte Versuchsanordnung ausgehend vom ursprünglichen Kerzenproblem) brauchte die Gruppe mit hoch ausgeprägter Fähigkeit zum kreativen Problemlösen nur 20 Sekunden um die Lösung zu finden. Neben dieser Standardbedingung führte Hussy (1992) dann zusätzlich eine sogenannte Fixierungsbedingung ein. Hierbei mussten die Vpn vor dem eigentlichen Experiment die Reissnägel mit einer Wäscheklammer in die Schachtel legen. Dadurch wurde die Fixierung - nämlich dass die Schachtel funktional als Behälter für die Reissnägel in dem Fall fungiert - verstärkt. Wie reagierten die beiden Gruppen von Vpn? Hussys Vermutung zufolge, war zu erwarten, dass Vpn mit hoher Fähigkeit zum kreativen Problemlösen einen Vorteil hätten. Die Erwartungen bestätigten sich auch hier. Die Fixierungsbedingung verlängerte die Lösungszeit, wobei dies vor allem bei den Personen mit geringer Fähigkeit zum kreativen Problemlösen zu beobachten war.

Insgesamt wiesen diese Ergebnisse also darauf hin, dass beim kreativen Denken, Fixierungen schneller und leichter gelöst, und nicht in gleicher Stärke ausgebildet werden. Auf den Übersetzungsprozess übertragen, kann man sich hier die Frage stellen, wie denn solche Fixierungen aussehen und wie sie gelöst werden können. Eine Möglichkeit der Auflösung von Fixierungen im Übersetzungsprozess (in Form von Denkblockaden) besteht in sogenannten Ablenkungsmanövern. So hat Kussmaul im Verlauf seiner Versuchsreihen anhand der Methode des Lauten Denkens und Beobachten der Vpn herausfinden können, dass Denkblockaden überwunden und besonders kreative Übersetzungsvorschläge nebst anderen Faktoren besonders dann hervorgebracht wurden, wenn die Vpn etwas ganz Anderes, Unerwartetes taten, wie z. B. aus dem Fenster schauen, sich im Kühlschrank etwas zum Essen holen gehen.

\subsubsection{Kreatives Problemlösen und unbewusste Prozesse}

\section{Bisoziationsmodell}

Wie in der Bisoziationstheorie von Koestler $(1964)^{9}$ können auch im Übersetzungsprozess unbewusste Verarbeitungsprozesse angenommen werden, die die Lösungssuche auch dann vorantreiben, wenn eine Person gerade nicht bewusst an einem Problem arbeitet. Die Lösungssuche beim kreativen Problemlösen erfolgt, so Hussy (2004), durch das freie Verknüpfen der Informationen (Bisoziation), bis gelegentlich die Lösung gefunden wird. Dazu kann es sowohl im Schlaf- als auch im Wachzustand kommen, wenn man sich mit dem Problem nicht bewusst beschäftigt, also in einer Art Pause. Erfolgreiche Bisoziationen gelangen ins Bewusstsein und vermitteln dort häufig den Eindruck, als hätte man die Lösung ohne eigenes Zutun gefunden. Nach Koestler sind die unbewussten Prozesse, so wie sie sich z. B. im Traum manifestieren, sehr viel flexibler als die bewussten Abläufe: Es fehle eine strenge Erfolgskontrolle, es fehle die Zielgerichtetheit und es fehle die Fixierung auf die Realität, die das bewusste Denken einengen. ${ }^{10}$ 


\subsubsection{Kreatives Problemlösen und automatisierte Prozesse}

Um den unmittelbaren Einfluss automatisierter Prozesse auf die Lösungsfindung zu illustrieren, auch hier wieder, ein Experiment von Hussy (1992): Die Vpn hatten Anagramme zu bearbeiten (KIMSU). In der Experimentalgruppe wurden diese Anagramme dadurch unbemerkt voraktiviert, dass den Vpn vorher Bilder gezeigt wurden, die die Lösungswörter enthielten (Musikladen). In der Kontrollgruppe hingegen konnten diese Lösungswörter durch eine geringe Perspektivenverschiebung nicht mehr gesehen werden, weshalb somit auch keine Voraktivierung der Anagramme erfolgte $^{11}$. Danach waren die Anagramme zu lösen. Die Experimentalgruppe benötigte eine signifikant kürzere Lösungszeit für die Bearbeitung der Anagramme als die Kontrollgruppe (13.7 sec vs. $30.2 \mathrm{sec}$.). In einer anschliessenden Befragung der Vpn konnte fest- und sichergestellt werden, dass keine unter ihnen den Zusammenhang zwischen den beiden Untersuchungsteilen (Bildbeschreibung, Anagrammlösung) bemerkt hatte. Keine der Beschreibungen enthielt zudem das Lösungswort. „Zweifellos beeinflussen“ - laut Hussy (idem.) und wie diese Versuchsergebnisse gleichzeitig zeigen - „also automatisierte Prozesse den bewussten (kontrollierten) Problemlösevorgang. " Hussy (idem.) weiter:

„Damit treten sie (die automatisierten Prozesse) zwangsläufig auch beim kreativen Problemlöseprozess auf. So manche kreativ erscheinende Problemlösung könnte - vermittelt über automatisierte Prozesse - zufällig zustande gekommen bzw. nahegelegt worden sein. Vielleicht hat Kekulé vor seiner entscheidenden Idee gerade über Schlangen gelesen bzw. die Abbildung einer Schlange bewusst oder unbewusst gesehen. Interessanterweise erklären solche automatisierten Prozesse auch den subjektiven Eindruck, oft nicht zu wissen, woher der entscheidende Einfall kam.“

Zusammenfassend kann gesagt werden, dass kreatives Problemlösen generell ein Spezialfall des allgemeinen Problemlösens ist. Kreatives Problemlösen nimmt vor allem in Situationen, in denen gängige Denk- und Vorstellungsweisen nicht zielführend sind, an Bedeutung zu, doch ebenso wie der allgemeine Problemlösevorgang setzt sich auch ein kreativer Prozess - Hussys Auffassung entsprechend - aus intentionalen, zielgerichteten, rationalen und logischen Anteilen zusammen. Es gibt heute noch zu wenige objektive Hinweise auf eine klare Trennung zwischen allgemeinem und kreativem Problemlösen, jedoch wollen wir es ausgehend von diesen Ausführungen ausgehend zumindest versuchen, kreativem Problemlösen im Übersetzungsprozess auf die Spur zu kommen, und sei es nur, um mehr darüber zu erfahren, dank welchen vielleicht anderen Denkprozessen wir auf die Bildung von alternativen Übersetzungsvorschlägen kommen, unter denen wir dann jeweils individuell die uns am passendsten, geeignetsten, am adäquatesten oder äquivalentesten erscheinende Lösung auswählen.

\section{BEOBACHTUNG UND BESREIBUNG KREATIVER MOMENTE IM ÜMBERTZUNGSPROZESS - ZWEI VERSUCHSREIHEN MIT TRANSLOG $2000^{12}$}

\subsection{Untersuchungsgegenstand, Zielsetzung und Hypothesen:}

Im vorliegenden Beitrag geht es nun darum mehr über mentale kreative Übersetzungsprozesse zu erfahren. Konzentrieren wollen wir uns aber insbesondere auf die Beobachtung und Beschreibung kreativer Momente im Übersetzungsprozess. Diese Momente bringen jeweils einen oder mehrere kreative Übersetzungsvorschläge 
hervor. Anhand dieser Vorgehensweise wollen wir im weiteren Verlauf auch mehr Erkenntnisse im Zusammenhang mit Problemlösungsstrategien gewinnen, die eben zu solchen kreativen Lösungen führen.

$\mathrm{Zu}$ aller erst war es jedoch erforderlich, generell mehr über den(die) Übersetzungsprozess(e) selbst, anders ausgedrückt über die einzelnen ihn/sie zusammensetzenden Phasen in Erfahrung zu bringen, wobei uns vor allem interessierte, in welche Phasen (in Anlehnung an bereits bestehende Modelle im Rahmen der Übersetzungswissenschaft) der Übersetzungsprozess aufgeteilt werden kann und wie sich diese Phasen zeitlich von einander unterscheiden. Mit anderen Worten, interessierte es uns, die genaue Länge der Phasen im Verhältnis zur gesamten, im Prozess benutzten Zeit zu ermitteln.

\subsubsection{Hypothese}

So wollten wir in einem ersten Schritt u. a. ermitteln, wie lang im Durchschnitt die Einarbeitungsphase vor der eigentlichen Übersetzung eines Textes ist, die eigentliche Übersetzungsphase oder die abschliessende Revisionsphase. Die zeitlichen Komponenten wurden dann jeweils mit der Qualitätsvariablen, bzw. der Kreativitätsvariablen in Verbindung gesetzt. Ziel dieses Vorhabens war es herauszufinden, ob eine längere Einarbeitungsphase bzw. eine längere Revisionsphase (im Vergleich jeweils $\mathrm{zu}$ einer daraus resultierenden kürzeren Verlaufsphase) zu einer besseren Übersetzungsleistung führt, d. h. zu einerseits qualitativ besseren Übersetzungen und andererseits kreativeren Lösungsvorschlägen. Damit wollten wir eine Gegenhypothese zu Gyde Hansens Beobachtung aufstellen, die im Rahmen einer Untersuchung an vier Studenten ${ }^{13}$ zu folgendem Ergebnis kam: „Lange Vorlaufsphasen sind keine Garantie für die Qualität der Übersetzungsprodukte“. (2002: 30). Erstens glauben wir nicht, dass eine Untersuchung an so wenigen Versuchspersonen als repräsentativ und statistisch signifikant gelten kann und sind der Ansicht, dass das Experiment schon allein aus diesem Grund wiederholt werden sollte. Weiters vermuteten wir stark - und diese Annahme liess sich v. a. auf Erfahrungswerte aus dem Übersetzungsunterricht zurückführen (Kommentare der Studenten, wie lange sie zu Hause für die jeweilige Übersetzung gebraucht haben und wofür sie die meiste Zeit in Anspruch genommen haben) - dass, eher genau das Gegenteil eintreffen müsste, nämlich, dass längere Einarbeitungs- und/oder Revisionsphasen zu einem besseren Übersetzungsergebnis führen. Eine mögliche Erklärung, sollte sich diese Hypothese als richtig herausstellen, ist vielleicht darin $\mathrm{zu}$ sehen, dass ein Übersetzer sowohl $\mathrm{zu}$ Beginn (während der Einarbeitungs- oder Orientierungsphase) als auch am Ende einer Übersetzung (während der Revisionsphase) eine globalere Herangehensweise an den Text und ein makrostrategischeres Verhalten hat, als während der eigentlichen Transferphase, in welcher er sich eher auf Punktuelles konzentriert und mikrostrategische Entscheidungen zur Überwindung von Übersetzungsproblemen trifft.

Die Überprüfung dieser Hypothese, genauer gesagt, die zeitliche Berechnung der einzelnen Phasen des Übersetzungsprozesses erfolgte per Translog 2000. Dank dieser Software ist es möglich objektive, nachvollziehbare und für andere auch nachprüfbare quantitative Daten zu gewinnen. 


\subsubsection{Hypothese}

Im weiteren Verlauf der Untersuchung sollte festgestellt werden, in welcher der drei Phasen des Übersetzungsprozesses die kreativen Übersetzungsvorschläge hervorgebracht werden. $\mathrm{Zu}$ Beginn der Untersuchung vermuteten, dass die meisten guten Lösungen in der Revisionsphase entstehen, nämlich nach längerem und intensivem Reflektieren (Reflexionsphasen bzw. Phasen, in denen die Versuchspersonen zögern oder zweifeln, können an den vom System aufgezeichneten Pausen erkannt werden obwohl nicht behauptet werden kann, dass die Versuchsperson eine Pause immer zum Nachdenken benutzt; während dieser Zeit kann auch anderes geschehen, z. B. Ablenkungsmanöver wie aus dem Fenster schauen, vor sich hin starren usw.), die meisten kreativen Lösungen im Vergleich dazu theoretisch jederzeit, v. a. aber auf die zwei letzten Phasen (Verlaufs- und Revisionsphase) verteilt entstehen können.

\subsubsection{Hypothese}

Schliesslich sollte noch überprüft werden, $o b$ der erste spontane Übersetzungsvorschlag, wie immer wieder vermutet, nicht etwa der kreativste und vielleicht sogar der beste ist. Es ist sicherlich jedem von uns schon einmal passiert, einen Übersetzungsvorschlag parat zu haben, diesen zu verwerfen, auf einen anderen bzw. vielleicht sogar auf mehrere andere Alternativvorschläge zu kommen, um in der Folge auf Umwegen wieder auf den ersten Vorschlag zurückzukommen und diesen als definitive Lösung zu verwenden. Diese Hypothese lässt sich wieder anhand unserer Translog-Protokolle verifizieren oder falsifizieren, genauer gesagt anhand der von den Übersetzern vorgenommenen Korrekturen und Revisionen.

\subsection{Methodologie}

\section{Beschreibung der Untersuchungsmethode: Arbeiten mit Translog}

Wie von Jakobsen (2002, 9ff; 151ff.) und dem Computerexperten Lasse Schou, den beiden Erfindern, beschrieben wird, können mit der Software Translog sämtliche messbare und vom System erfassbare, quantitative Daten festgehalten werden. Der Schreibprozess der Versuchsperson wird mit Translog zeitlich genau registriert und sämtliche Tastaturbewegungen (Pausen, Korrekturen, Curserbewegungen usw.) beim Lesen des AT oder beim Verfassen des ZT festgehalten ${ }^{14}$.

\subsubsection{Beschreibung des Versuchsdesigns}

Versuchspersonen waren insgesamt 40 Studentinnen und Studenten der deutschen Abteilung aus dem 1. und/oder 2. Studienabschnitt der Ecole de Traduction et d'Interprétation der Universität Genf. Ihre Muttersprache ist Deutsch und sie wurden in beiden Versuchsreihen gebeten, jeweils einen Text aus dem Französischen bzw. Italienischen ins Deutsche zu übersetzen. Die erste Versuchsreihe (Pilot) fand im April 2003 statt, die zweite Versuchsreihe zur Kontrolle im November 2003 ${ }^{15}$. Der Text wurde im Hinblick auf Schwierigkeit und Länge vom Versuchsleiter so ausgewählt, dass er leicht in zwei Stunden übersetzt werden konnte. Dies ist deshalb wichtig zu erwähnen, da in anderen Versuchsreihen, in denen Translog zum Einsatz kam, 
es z. B. darum ging festzustellen, wie Übersetzer mit unterschiedlicher Erfahrung (Studenten, professionnelle Übersetzer) unter Zeitdruck ${ }^{16}$ arbeiten und wie sich der Faktor Zeit u. U. auch als Stressfaktor auf die Qualität des Produkts auswirkt ${ }^{17}$. Nachdem der Schwerpunkt unserer Untersuchung aber ganz anders gelagert ist, war es wichtig, dass unsere Vpn in ihrer Arbeitsweise nicht durch den Faktor Zeit bzw. eine andere Limite beeinflusst gewesen wären.

\section{Beschreibung der Evaluierung der Übersetzungen - Auflistung der Kriterien}

Bei einer Untersuchung des kreativen Problemlösens beim Übersetzen, zunächst ausgehend von den jeweiligen Produkten (auf den Prozess werden wir uns später konzentrieren), müssen in einem ersten Schritt kreative Leistungen als solche erkannt und von nicht kreativen Leistungen unterschieden bzw. abgehoben werden. Zu diesem Zweck müssen die einzelnen Übersetzungen aufs erste einmal beurteilt ${ }^{18}$ werden und dies nach möglichst objektiven, für andere nachvollziehbar und nachprüfbaren Kriterien, die auch Wiederholungen der Experimente und Vergleiche mit anderen Versuchsreihen in Zukunft ermöglichen sollten.

Die Übersetzungen wurden in diesem Sinne also somit einerseits auf Fehler hin korrigiert, andererseits wurden aber auch pragmatisch, semantisch, stilistisch und idiomatisch gute sowie kreative Übersetzungsvorschläge in die Bewertung mit einbezogen. Es handelte sich um eine offene „Evaluierungskriterienliste“, die aber keinesfalls Anspruch auf Vollständigkeit erhebt. Bei der Beschreibung der Fehlertypen stützten wir uns v. a. auf Gyde Hansens Fehlertypologie.

Positiv bewertet wurden die folgenden beiden Kriterien:

1. Kreativität: Wenn der hervorgebrachte Übersetzungsvorschlag eine neue (noch nie dagewesene, nicht konventionelle), originelle (unerwartete) und angemessene Lösung darstellt (in dem Sinne, als dass sie als qualitativ gute und kreative Leistung dem Übersetzungszweck und den Erwartungen des Zielpublikums entsprechend auch von anderen Beurteilern als kreativ und geeignet akzeptiert würde).

2. Qualität (vgl. zur Beurteilung von Übersetzungsleistungen, auch Hannelore LeeJahnke, 2001: 258ff)

- Skopos: Der Übersetzungsvorschlag muss dem vom Autor in der AS intendierten Zweck entsprechen und diesen in der ZS auch weiterhin erfüllen (Funktionsvarianz).

- Idiomatik: Besonders idiomatische Ausdrücke, Formulierungen und Wendungen.

- Stilistik: besonders gute stilistische Lösungen

- Geeignete Verwendung von Übersetzungsstrategien wie intentierte Straffungen / Etoffements, Erweiterungen, Auslassungen usw.

\subsection{Analyse und Synthese der Ergebnisse aus den beiden Versuchsreihen}

\subsubsection{Zu den einzelnen Phasen des Übersetzungsprozesses}

Ergebnisse aus beiden Versuchsreihen im Hinblick auf ein bestimmtes

Verhaltensmuster bei der Verteilung der Phasen unter Angabe der Zeit

Schritt 1: Analyse der Phasen im Hinblick auf Verhaltensmuster in den Übersetzungsprozessen der 40 Versuchspersonen (in der zweiten Versuchsreihe übersetzten 3 davon in zwei Sprachrichtungen, weshalb dies hier insgesamt 26 Testergebnisse ergab) aus der ersten Versuchsreihe mit Translog (Pilotprojekt), Versuchsreihe I, die wir im April 2003 vorgenommen hatten. 
Schritt 2: Kontrollversuche dazu, in einer neuen Versuchsreihe mit Translog, Versuchsreihe 2, im November 2003.

Verteilung der Phasen mit Zeitangaben (Versuchsreihe 1) für die Übersetzungsrichtung FR/DE:

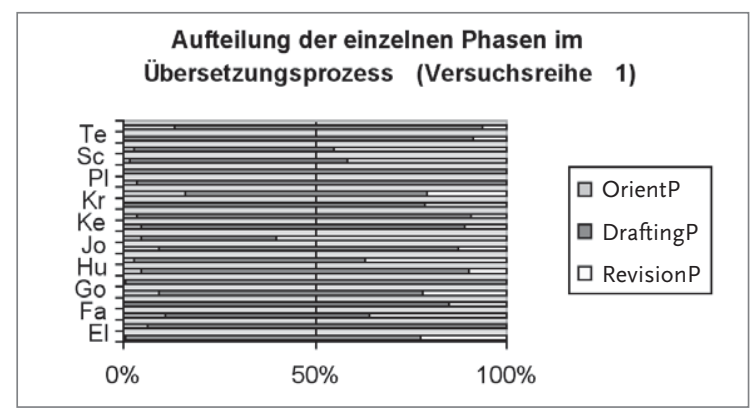

\begin{tabular}{|l|r|r|r|}
\hline & OP & DP & RP \\
\hline El & 0.15 & 24.5 & 7.06 \\
\hline Er & 2.08 & 29.3 & 0 \\
\hline Fa & 4.36 & 21.4 & 14.54 \\
\hline Gie & 0.06 & 23.08 & 4.15 \\
\hline Go & 3.44 & 26.41 & 8.31 \\
\hline $\mathrm{Ha}$ & 0.17 & 39.33 & 0 \\
\hline $\mathrm{Hu}$ & 1.07 & 20.44 & 2.29 \\
\hline Ja & 1.06 & 21.28 & 13.18 \\
\hline Joh & 4.02 & 33.11 & 5.46 \\
\hline Jol & 2.08 & 16.01 & 27.15 \\
\hline
\end{tabular}

\begin{tabular}{|l|r|r|r|}
\hline $\mathrm{Ke}$ & 1.38 & 26.22 & 3.36 \\
\hline $\mathrm{Ko}$ & 1.14 & 30.57 & 3.30 \\
\hline $\mathrm{Kr}$ & 0 & 28.06 & 7.54 \\
\hline $\mathrm{Lu}$ & 4.12 & 16.35 & 5.35 \\
\hline $\mathrm{Pl}$ & 1.42 & 36.52 & 0 \\
\hline $\mathrm{Ra}$ & 0 & 37.20 & 0 \\
\hline $\mathrm{Sch}$ & 0.58 & 16.25 & 12.03 \\
\hline $\mathrm{Schr}$ & 1.06 & 17.35 & 15.14 \\
\hline $\mathrm{Te}$ & 0.09 & 33.54 & 3.19 \\
\hline $\mathrm{Tr}$ & 5.26 & 31.25 & 2.50 \\
\hline Gesamtsumme & 33.54 & 528.17 & 134.55 \\
\hline pro VP & 1.677 & 26.41 & 6.73 \\
\hline
\end{tabular}

Legende: $\mathrm{OP}=$ Orientationphase, $\mathrm{DP}=$ Draftingphase, $\mathrm{RP}=$ Revisionphase

Im Schnitt wurden pro Versuchsperson 1.7 min für die Orientierungsphase, $26.4 \mathrm{~min}$ für die Verlaufsphase sowie 6.7 min für die Revisionsphase verwendet, woraus hervorgeht, dass die meiste Zeit - wie erwartet - für den eigentlichen Transfer, hier Verlaufsphase genannt, aufgebracht wurde.

Die durchschnittliche Dauer des gesamten Prozesses betrug bei diesem Text 34.8 min, wobei die am längsten arbeitende Versuchsperson 45.2 min mit der Übersetzung verbrachte und die schnellste Übersetzung in nur weniger als der Hälfte der Zeit, nämlich in 24.2 min angefertigt wurde.

Interessant war festzustellen, dass zwei Personen überhaupt keine Orientierungsphase eingelegt und sofort mit dem Schreibprozess begonnen haben (siehe dazu die Daten von $\mathrm{Kr}$ sowie $\mathrm{Ra}$ ) und drei andere Personen eine ganz minimale Dauer von unter bzw. gleich 0.17 min aufweisen (El, Ha und Te).

Was die Revisionsphase anbelangt, so ist aufgefallen, dass diese bei gleich vier Personen (Er, Ha, Pl und wieder Ra) ganz ausfällt, mit anderen Worten, dass diese Personen nachdem sie den Text zu Ende übersetzt hatten, keine einzige Revision 
mehr vorgenommen haben, wobei wir natürlich nicht ausschliessen können, dass Korrekturen vielleicht bereits während der Verlaufsphase gleich oder rückwirkend angebracht worden sind.

Auffallend ist weiters der Prozess der Studentin Jol, deren Revisionsphase zum einen einmal deutlich länger andauerte als die ihrer Komilitonninen und zum anderen auch länger war als die Verlaufsphase selbst. Zur Orientierung benötigte sie 2.08 min, zum eigentlichen Transfer 16.01 und für Revisionen beachtliche $27.5 \mathrm{~min}$.

Deutlich über dem Durchschnittswert der Revisionsphase pro Person bei 6.73 min liegen auch die Werte zweier anderer Studenten: Schr verwendete 15.14 min und Fa 14.54 min für Revisionen danach.

Welche Auswirkungen diese Beobachtungen auf den Prozess, genauer gesagt, auf die Kreativität bei der Übersetzung gehabt haben, wird sich zeigen, wenn wir die Prozessvariablen mit dem Kreativitätsfaktor in Bezug stellen werden (Überprüfung im Rahmen der Hypothese 1). Im Vergleich und zur Kontrolle dieser Beobachtungen vorerst aber noch die Ergebnisse aus der Versuchsreihe 2:

Verteilung der Phasen mit Zeitangaben (Versuchsreihe 2) F/D:

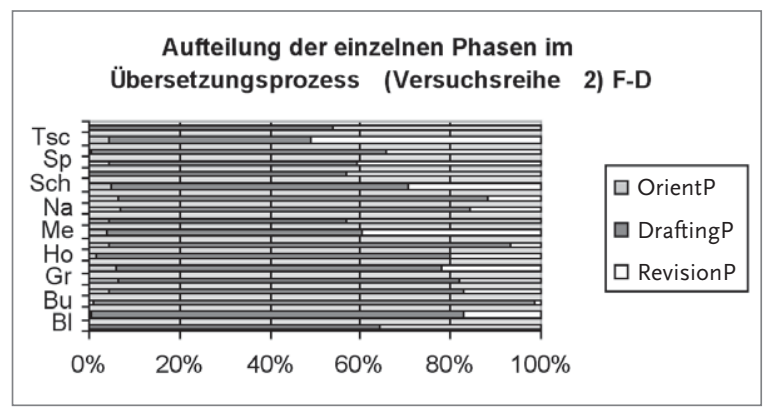

\begin{tabular}{|l|r|r|r|l|r|r|r|}
\hline Name & OP & DP & RP & Name & OP & DP & RP \\
\hline $\mathrm{Bl}$ & 0.04 & 20.12 & 11.14 & Na & 2.31 & 26.96 & 5.45 \\
\hline $\mathrm{Br}$ & 0.08 & 22.21 & 4.58 & Pe & 1.01 & 13.37 & 1.89 \\
\hline $\mathrm{Bu}$ & 0.11 & 14.31 & 0.25 & Schweg & 2.17 & 29.16 & 13.02 \\
\hline $\mathrm{Gi}$ & 1.51 & 27.48 & 6.01 & Schwen. & 0.06 & 16.11 & 12.25 \\
\hline $\mathrm{Gr}$ & 2.56 & 30.04 & 7.21 & Sp & 1.59 & 21 & 15.42 \\
\hline $\mathrm{He}$ & 1.58 & 20.09 & 6.14 & Su & 0.09 & 23.11 & 12.19 \\
\hline $\mathrm{Ho}$ & 0.43 & 21.22 & 5.52 & Tsch & 1.28 & 12.5 & 14.41 \\
\hline $\mathrm{Kh}$ & 2.23 & 47.2 & 3.59 & Vo & 0.07 & 16.43 & 14.15 \\
\hline $\mathrm{Me}$ & 1.53 & 23.55 & 16.35 & TOTAL & 20.21 & 403.02 & 150.47 \\
\hline $\mathrm{Mo}$ & 1.56 & 18.16 & 15.05 & pro VP & 1.1 & 22.4 & 8.3 \\
\hline
\end{tabular}

Legende: $\mathrm{OP}=$ Orientationphase, $\mathrm{DP}=$ Draftingphase, $\mathrm{RP}=$ Revisionphase

Insgesamt macht die Orientierungsphase hier $1.1 \mathrm{~min}$ des Schreibprozesses aus, die Verlaufsphase 22.4 min und die Revisionsphase 8.3 min.

Durchschnittlich wurden für den gesamten Prozess bei diesem Text 33 min aufgebracht, wobei die schnellste Übersetzung in erstaunlichen $15.1 \mathrm{~min}$ und die am längsten andauernde in 54.4 min angefertigt wurden. 
Alle Studenten haben bei dieser Versuchsreihe sowohl eine Orientierungs- als auch eine Revisionsphase miteingelegt, zumindest war nicht mehr beobachtbar und/ oder feststellbar, dass diese, wie es im März 2003 der Fall war, ausgefallen wäre.

Interessante Prozesse weisen die Studenten Bu, Schw, Suund Vo auf. Alle vier charakterisiert eine äusserst knappe Orientierungsphase (Schw 0.06min, Vo 0.07min, $\mathrm{Su} 0.09 \mathrm{~min}$ und $\mathrm{Bu} 0.11 \mathrm{~min}$ ) und eine Studentin eine erstaunlich kurze Revisionsphase (dies ist der Fall bei Bu mit 0.25min).

Was andere Auffälligkeiten im Prozess anbelangt, so gibt es auch hier eine Studentin, deren Revisionsphase von der Dauer her über der Verlaufsphase liegt. Es handelt sich um die Studentin Tsch, deren Orientierungsphase $1.28 \mathrm{~min}$ (leicht über dem Durchschnittswert von 1.1min), die Verlaufsphase 12.5 min (deutlich unter dem Durchschnittswert von 22.4min) und deren Revisionsphase bei $14.41 \mathrm{~min}$ (klar über dem Durchschnittswert von 8.3min) liegt.

Und auch in dieser Versuchsreihe zeichnen sich wieder gewisse Studenten durch eine überdurchschnittlich (der Durchschnittswert pro Person liegt bei $8.3 \mathrm{~min}$ ) lange andauernde Revisionsphase aus (siehe Me $16.35 \mathrm{~min}$, Sp $15.42 \mathrm{~min}$, Mo $15.05 \mathrm{~min}$, Tsch $14.41 \mathrm{~min})$.

Auch hier wollten wir in Erfahrung bringen, welche Konsequenzen diese Beobachtungen im Endeffekt auf die Kreativität der Übersetzung selbst haben ${ }^{19}$.

Für das Sprachenpaar Italienisch-Deutsch gestaltete sich die Verteilung der einzelnen im Übersetzungsprozess vorkommenden Phasen wie folgt:

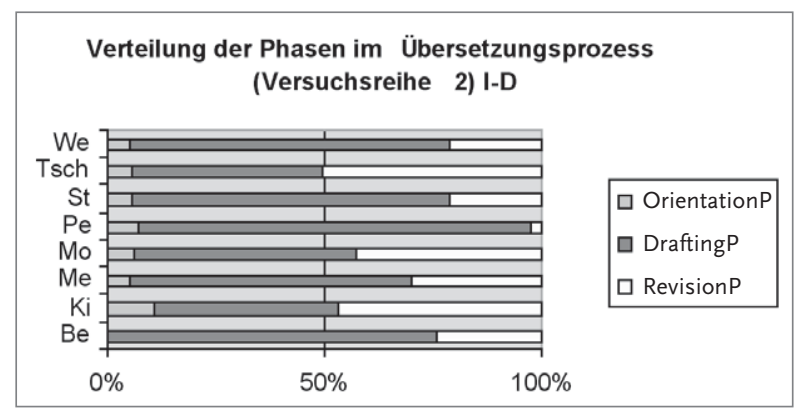

\begin{tabular}{|l|r|r|r|}
\hline Name & OP & DP & RP \\
\hline $\mathrm{Be}$ & 0.05 & 28.16 & 9.02 \\
\hline $\mathrm{Ki}$ & 3.47 & 13.37 & 15.01 \\
\hline $\mathrm{Me}$ & 2.37 & 28.24 & 13.2 \\
\hline $\mathrm{Mor}$ & 2.25 & 17.77 & 15.03 \\
\hline $\mathrm{Pe}$ & 1.41 & 17.33 & 0.48 \\
\hline $\mathrm{St}$ & 2.03 & 25.52 & 7.3 \\
\hline $\mathrm{Tsch}$ & 1.43 & 11.57 & 13.16 \\
\hline $\mathrm{We}$ & 1.52 & 22.09 & 6.25 \\
\hline TOTAL & 14.53 & 164.05 & 79.45 \\
\hline pro VP & 1.8 & 20.5 & 9.9 \\
\hline
\end{tabular}

Legende: $\mathrm{OP}=$ Orientationphase, $\mathrm{DP}=$ Draftingphase, $\mathrm{RP}=$ Revisionphase

Im Durchschnitt wurden bei diesem Text für die Orientierungsphase 1.8 min verwendet, für die Verlaufsphase 20.5 min und für die Revisionsphase $9.9 \mathrm{~min}$. 
Im Durchschnitt dauerte der gesamte Prozess bei der Übersetzung aus dem Italienischen $32.2 \mathrm{~min}$, was dem Ergebnis für die Übersetzung aus dem Französischen bei der gleichen Textmenge in etwa gleichkommt (33 min). Besonders auffallend ist auch hier wieder die Tatsache, dass eine Studentin (Be $0.05 \mathrm{~min}$ ) fast keine Orientierungsphase aufweist ${ }^{1}$ und ein Student eine überaus knappe Revisionsphase (es handelt sich hier um Pe mit 0.48min).

Interessant war ausserdem festzustellen, dass es auch hier wieder vorgekommen ist, dass die Revisionsphase länger sein kann als der eigentliche Transfer. Durchaus auffällig, war zu sehen, dass es sich bei einem der beiden Fälle um dieselbe Studentin handelte, wie in der Übersetzungskombination FR/DE. Sie (vgl. Daten von Tsch) verwendete 1.43 min für die Orientierungsphase, 11.57 min für die Verlaufsphase und 13.16 min für die Revisionsphase. Aus dem Unterricht wissen wir, dass es sich hier um eine ausgezeichnete Studentin mit gleichbleibend erfreulichem Leistungspotenzial handelt und spätestens an dieser Stelle drängte sich uns erneut die Vermutung auf, dass eine längere Revisionsphase vielleicht ein Indiz für qualitativ gute und u. U. sogar kreative Übersetzungen ist. Den zweiten im Unterschied zu den anderen Studenten abweichenden Prozess weist der Student Ki auf, dessen Revisionsphase auch über der Dauer der Verlaufsphase lag. Er verwendete 3.47 min zur Orientierung, 13.37 min für den Transfer und $15.01 \mathrm{~min}$ für Revisionen nachdem der Text einmal ganz zu Ende übersetzt war ${ }^{20}$.

Wie schon bereits mehrmals angekündigt, sollen die zeitlichen Komponenten nun mit dem Kreativitätsfaktor in Verbindung gesetzt werden, um herauszufinden ob eine längere Einarbeitungsphase bzw. eine längere Revisionsphase (im Vergleich jeweils zu einer kürzeren Übersetzungsphase) effektiv auch zu kreativeren Übersetzungsleistungen führt ${ }^{21}$.

\subsubsection{Korrelation der einzelnen Phasen des Übersetzungsprozesses untereinan- der und Auswirkungen auf Kreativität}

\section{Korrelation Orientierungs- bzw. Revisionsphase in Bezug zur Kreativität Versuchsreihe 2}

Für das Übersetzungspaar IT/DE war auch in der Versuchsreihe 2 keine Gesetzmässigkeit zwischen Orientierungsphase und Kreativität diagnostizierbar. Somit kann fürs erste auch nicht postuliert werden, dass eine längere Einarbeitungsphase zu kreativeren Übersetzungen führe: zwar liegt die Orientierungsphase bei denjenigen Übersetzungen, die kreative Lösungen enthalten - als Beispiel seien hier die Ergebnisse der Studenten Mo, Me und Ki genannt - über dem Durchschnittswert von $1.8 \mathrm{~min}$, jedoch weist diejenige Übersetzung, die die meisten kreativen Vorschläge enthielt (Tsch), eine relativ kurze Orientierungsphase auf (mit 1.43 min liegt sie unter dem Durchschnittswert). Zur Überprüfung dieses Ergebnisses sollen uns die Übersetzungsleistungen aus dem Sprachenpaar FR/DE dienen. 


\begin{tabular}{|l|r|r|}
\hline Name & OP & K \\
\hline $\mathrm{Be}$ & 0.05 & 0.0 \\
\hline $\mathrm{Pe}$ & 1.41 & 1.5 \\
\hline Tsch & 1.43 & 6.0 \\
\hline $\mathrm{We}$ & 1.52 & 0.0 \\
\hline $\mathrm{St}$ & 2.03 & 0.0 \\
\hline $\mathrm{Mo.}$ & 2.25 & 1.5 \\
\hline $\mathrm{Me}$ & 2.37 & 1.5 \\
\hline $\mathrm{Ki}$ & 3.47 & 1.5 \\
\hline
\end{tabular}

\begin{tabular}{|l|l|l|}
\hline Name & OP & K \\
\hline $\mathrm{Bl}$ & 0.04 & 0.0 \\
\hline Schwen & 0.06 & 1.5 \\
\hline $\mathrm{Vo}$ & 0.07 & 0.0 \\
\hline $\mathrm{Br}$ & 0.08 & 0.0 \\
\hline $\mathrm{Su}$ & 0.09 & 0.0 \\
\hline $\mathrm{Bu}$ & 0.11 & 0.0 \\
\hline $\mathrm{Ho}$ & 0.43 & 0.0 \\
\hline $\mathrm{Pe}$ & 1.01 & 0.0 \\
\hline $\mathrm{Tsch}$ & 1.28 & 3.0 \\
\hline $\mathrm{Gi}$ & 1.51 & 4.5 \\
\hline $\mathrm{Me}$ & 1.53 & 3.0 \\
\hline $\mathrm{Mo}$ & 1.56 & 0.0 \\
\hline $\mathrm{He}$ & 1.58 & 0.0 \\
\hline $\mathrm{Sp}$ & 1.59 & 1.5 \\
\hline $\mathrm{Schweg}$ & 2.17 & 1.5 \\
\hline $\mathrm{Kha}$ & 2.23 & 1.5 \\
\hline $\mathrm{Na}$ & 2.31 & 3.0 \\
\hline $\mathrm{Gr}$ & 2.56 & 0.0 \\
\hline
\end{tabular}

Die kreativen Übersetzungen liegen zwar fast alle (abgesehen von Schw mit einem Kreativitätsfaktor von 1.5 und einer Orientierungsphase von $0.06 \mathrm{~min}$ ) in der oberen Hälfte der im Schnitt für die Orientierung aufgewandte Zeit (siehe Tsch, Gi, Me, Na aber auch Sp, Schweg und Kh), jedoch war auch hier nicht eindeutig feststellbar, dass eine längere Orientierungsphase zu kreativeren Übersetzungen führt.

Es kann somit schlussgefolgert werden, dass zwar eine leichte Tendenz dahingehend besteht, dass längere Orientierungsphasen im Übersetzungsprozess zu kreativeren Übersetzungsleistungen führen, dies jedoch keine absolute Regel darstellt.

Nun zu einer eventuellen Korrelation zwischen der Revisionsphase und dem Kreativitätsaspekt. Auch hier wollen wir unsere Ausgangshypothese überprüfen und herausfinden, ob eine längere Revisionsphase zu kreativeren Übersetzungen führt oder nicht. Dazu haben wir die beiden Variablen wieder in Bezug zueinander gesetzt und eine gewisse Tendenz festzustellen versucht.

Wie aus den untenstehenden Zahlen für das Übersetzungspaar IT/DE ersichtlich wird, hat sich unsere Ausgangshypothese als richtig herausgestellt. Diejenigen Studenten, die im Vergleich zu den anderen eine deutlich längere Revisionsphase haben, bringen es auch zu kreativeren Übersetzungsleistungen.

\begin{tabular}{|l|r|r|}
\hline Name & RP & K \\
\hline Pes & 0.48 & 1.5 \\
\hline Weh & 6.25 & 0.0 \\
\hline Sta & 7.3 & 0.0 \\
\hline Ber & 9.2 & 0.0 \\
\hline
\end{tabular}

\begin{tabular}{|l|r|r|}
\hline Tsch & 13.16 & 6.0 \\
\hline $\mathrm{Me}$ & 13.2 & 1.5 \\
\hline $\mathrm{Ki}$ & 15.01 & 1.5 \\
\hline $\mathrm{Mo}$ & 15.03 & 1.5 \\
\hline Durchschnitt & 10.0 & \\
\hline
\end{tabular}


An dieser Stelle drängt sich natürlich sofort die Frage auf, ob die in den Übersetzungen enthaltenen kreativen Lösungsvorschlägen etwa in der Revisionsphase entstehen. Dieser Frage wollen wir an anderer Stelle nachgehen (siehe Ausgangshypothese Nr. 3). Zunächst wollen wir unsere letzte Beobachtung anhand dem Übersetzungspaar FR/DE (Versuchsreihe 2) erneut überprüfen:

\begin{tabular}{|l|r|r|}
\hline Name & RP & K \\
\hline $\mathrm{Bu}$ & 0.25 & 0.0 \\
\hline $\mathrm{Pe}$ & 1.89 & 0.0 \\
\hline $\mathrm{Kh}$ & 3.59 & 1.5 \\
\hline $\mathrm{Br}$ & 4.58 & 0.0 \\
\hline $\mathrm{Na}$ & 5.45 & 3.0 \\
\hline $\mathrm{Ho}$ & 5.52 & 0.0 \\
\hline $\mathrm{Gi}$ & 6.01 & 4.5 \\
\hline $\mathrm{He}$ & 6.14 & 0.0 \\
\hline $\mathrm{Gr}$ & 7.21 & 0.0 \\
\hline
\end{tabular}

\begin{tabular}{|l|c|c|}
\hline $\mathrm{Bl}$ & 11.14 & 0.0 \\
\hline $\mathrm{Su}$ & 12.19 & 0.0 \\
\hline Schwen & 12.25 & 1.5 \\
\hline Schweg & 13.02 & 1.5 \\
\hline Vo & 14.15 & 3.0 \\
\hline $\mathrm{Tsch}$ & 14.41 & 3.0 \\
\hline $\mathrm{Sp}$ & 15.42 & 1.5 \\
\hline $\mathrm{Mo}$ & 15.05 & 0.0 \\
\hline $\mathrm{Me}$ & 16.35 & 3.0 \\
\hline Durchschnitt & 9.15 & \\
\hline
\end{tabular}

Auch hier lässt sich erkennen, dass bei längerer Revisionsphase die kreativeren Übersetzungsleistungen entstanden sind. Jedoch stellt dies, wie sich anhand der Übersetzungen von $\mathrm{Na}$ und Gi feststellen lässt, keine Regel dar.

Abschliessend kann somit behauptet werden, dass längere Revisionsphasen ein starkes Indiz für kreative Übersetzungsleistungen sind, lange Revisionsphasen an sich jedoch noch keine Garantie für Kreativität darstellen.

Graphisch lassen sich diese Beobachtungen wie folgt darstellen:

\begin{tabular}{|l|c|c|c|}
\hline Name & OrientT & RevisionP & Creativity \\
\hline BlF & 0.04 & 11.14 & 0 \\
\hline Br F & 0.08 & 4.58 & 0 \\
\hline Bu F & 0.11 & 0.25 & 0 \\
\hline Gi F & 1.51 & 6.01 & 4.5 \\
\hline Gr F & 2.56 & 7.21 & 0 \\
\hline He F & 1.58 & 6.14 & 0 \\
\hline Ho F & 0.43 & 5.52 & 0 \\
\hline Kh F & 3.23 & 3.59 & 1.5 \\
\hline Me F & 1.53 & 16.35 & 3 \\
\hline MoF & 1.56 & 15.05 & 0 \\
\hline Na F & 2.31 & 5.45 & 3 \\
\hline PeF & 1.01 & 1.89 & 0 \\
\hline Schweg F & 2.17 & 13.02 & 1.5 \\
\hline
\end{tabular}

\begin{tabular}{|l|r|r|r|}
\hline Schwen F & 0.06 & 12.25 & 1.5 \\
\hline Spe F & 1.59 & 15.42 & 1.5 \\
\hline Su F & 0.09 & 12.19 & 0 \\
\hline Tsch F & 1.28 & 14.41 & 3 \\
\hline VoF & 0.07 & 14.15 & 0 \\
\hline BeI & 0.05 & 9.02 & 0 \\
\hline Kis I & 3.47 & 15.01 & 1.5 \\
\hline Me I & 2.37 & 13.2 & 1.5 \\
\hline Mo I & 2.25 & 15.03 & 1.5 \\
\hline Pe I & 1.41 & 0.48 & 1.5 \\
\hline StI & 2.03 & 7.3 & 0 \\
\hline Tsch I & 1.43 & 13.16 & 6 \\
\hline WeI & 1.52 & 6.25 & 0 \\
\hline
\end{tabular}




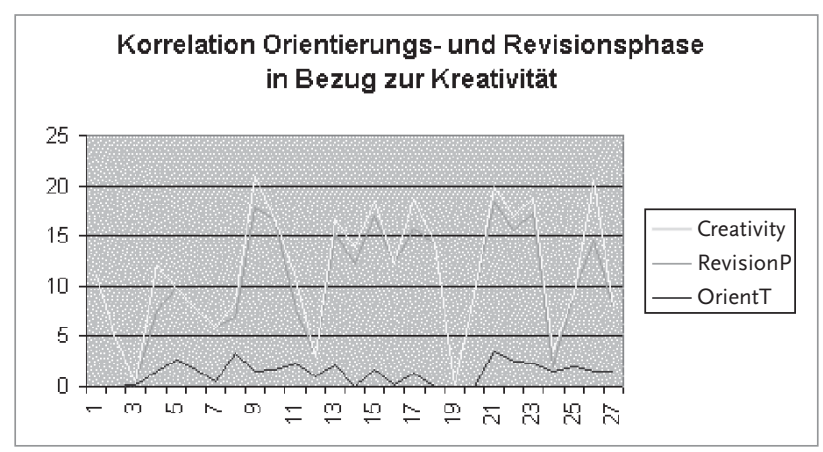

\section{Überprüfung der 2. Hypothese - Vorkommen kreativer Momente im Übersetzungsprozess}

In welcher der drei Phasen des?Übersetzungsprozesses werden nun kreative Übersetzungsvorschläge hervorgebracht? Auch auf diese beiden Fragen versuchten wir bereits eingangs in Form einer Hypothese zu antworten und vermuteten, dass die meisten guten Lösungen in der Revisionsphase entstehen, die meisten kreativen Lösungen jedoch jederzeit, auf die drei Phasen gleich verteilt, entstehen können.

Diese Vermutung gilt es nun anhand der vorhandenen Translog Protokolle zu verifizieren oder zu falsifizieren, wobei wir uns im Rahmen dieses Beitrags auf den hier im Mittelpunkt stehenden Kreativitätsaspekt konzentrieren wollen.

In der Versuchsreihe 2, die speziell zur Untersuchung des Kreativitäts-Begriffs herangezogen wurde, hat sich folgendes Bild ergeben:

Zur besseren Veranschaulichung, wie bei der Analyse und der Beantwortung dieser Frage vorgegangen wurde, soll uns hier als Musterübersetzung die von der Studentin Tsch angefertigte Arbeit dienen. In der Sprachenkombination IT/DE enthielt die Übersetzung dieser Studentin insgesamt 4 besonders kreative Lösungsvorschläge.

Es handelte sich dabei um die folgenden Stellen im AT und die jeweils darunter mitabgebildeten Übersetzungen:

AT: [...] Il rischio di cadere nel banale esiste, ma [1] al tempo stesso è innegabile che, come mette in luce [2] la sarcastica espressione di Longanesi, la famiglia sia l'istituzione su cui si fonda l'Italia [3].

ZT: [...] Auch wenn es etwas vereinfachend klingen mag [1.] die Wahrheit der sarkastischen Aussage von Longanesi lässt sich nicht abstreiten [2.] Die Familie ist zentral in Italien [3].

AT: E questo spiega da dove vengano lo scarso senso civico, l'arte di arrangiarsi [4], la sfiducia cronica nelle stato e nelle istituzioni che da secoli accompagnano gli italiani. [...]

ZT: Dieser Umstand erklärt das schwache Bürgerbewusstsein, die Kunst sich durchzuschlängeln [4.] und das chronische Misstrauen gegenüber dem Staat und den Ämtern, welches die Italiener schon seit Jahrhunderten hegen.

Nun stellt sich die Frage, in welcher Prozessphase die Studentin auf diese Lösungsvorschläge gekommen ist und, in diesem Zusammenhang von Interesse, ob es sich bei diesen definitiven Lösungsvorschlägen auch jeweils um die Erstversion handelt 
oder ob die Studentin, bevor sie auf diese Endlösungen gekommen ist, vielleicht auch schon andere Alternativvorschläge hatte, die sie in der Folge auf die definitiven Problemlösungen gebracht haben. Bei dieser Gelegenheit wollten wir nämlich gleichzeitig herausfinden, ob es für die jeweiligen kreativen Übersetzungsvorschläge bestimmte immer wiederkehrende Auslöser, wie z. B. Pausen oder die eben angesprochenen Revisionen gegeben hat. Sollte sich dies herausstellen und können dabei Regelmässigkeiten beachtet werden, müsste das Hauptaugenmerk in Zukunft vermehrt auf diese Auslöser gelegt werden, um generell mehr darüber zu erfahren, welche Faktoren zum Hervorbringen von kreativen Lösungsvorschlägen führen. Rein intuitiv interessieren wir uns im Augenblick (und dies ist auch für künftige, weiterführende Studien so geplant) für die Anzahl und Art der Pausen, sowie für die Anzahl und Art der verschiedenen Revisionen und Korrekturen während des gesamten Prozesses, da diese, wie wir meinen, aufschlussreiche Phänomene zum Hervorbringen von kreativen Lösungen sein könnten.

Mithilfe Tsch Logdatei wird ersichtlich, wie die kreativen Übersetzungsvorschläge zustande gekommen sind:

Ansatzweise schon in der Verlaufsphase von 08.05.97 - 11.40.43

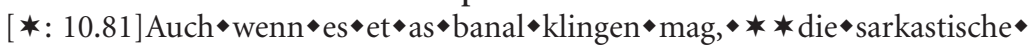

Aussage von $\bullet$ Longanesi $* \bullet[*: 27.41]^{* * *} *, * * * *$ Italien $\bullet$ beruht $\bullet$ auf $\bullet$ der

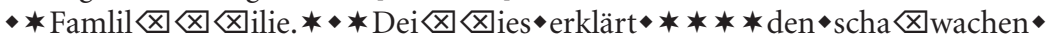

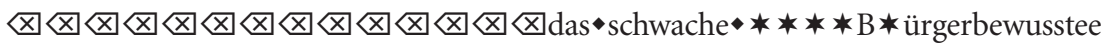

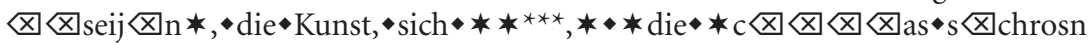

$\otimes \otimes$ nische $\bullet$ Misstrauen $*$ gegenüber $\bullet$ dem $\bullet$ Staat $\bullet$ und $\bullet$ den $*$ Ints $\otimes \otimes \otimes \otimes$

**** Ämtern, ** welches schon $* *$ seit

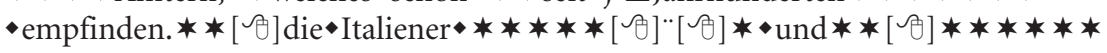

$* * * \bullet[*: 31.37]$

Der Rest in der Revisionsphase von 15.58.47 - 18.32.09

Grund $* \otimes c * *[\Theta] *[\vartheta] * \otimes * \bullet[*: 28.07][\vartheta] * *$ lässt $\bullet$ sich $\bullet$ nicht $\bullet$ abstreig

$凶 \operatorname{ten} * * *[\Theta] * *$ vereinfachend $* * * *[\Theta] \otimes *:[*: 14.42][\Theta] *$ ie $*[\Theta]$

$* \otimes * *[\vartheta] \mathrm{D} *[\vartheta] \bullet$ ist $*$ zentral $\bullet$ in $\bullet \mathrm{Ta} \otimes \otimes \operatorname{XItalien}[*: 14.41][\vartheta] *$ anzupassen

[*: 13.89][田]hege $\otimes * * * * * * * * *[\vartheta] \otimes * *[\Theta] \otimes \otimes * * * *[*: 10.69]$

Sowie in der Revisionsphase von $21.03 .41-22.05 .93$

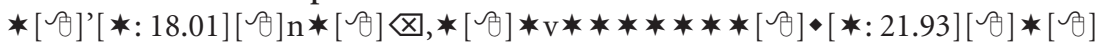

WAhrheit $\bullet \operatorname{der} *[\vartheta]$

\section{Erklärungen:}

Nach 08.05.97 Min. legt die Studentin eine Pause von 10.81 Sek. ein. Wahrscheinlich handelt es sich hier um eine Orientierungspause, die die Studentin dazu nützte zu überlegen, wie sie den nächsten Satz in Angriff nehmen soll. Jedoch können wir nicht mit Gewissheit sagen, ob sie in Gedanken wirklich schon beim nächsten Satz war. Vielleicht überlegte sie sich auch noch einmal etwas zum vorhergehenden Satz, vielleicht war sie mit ihren Gedanken auch ganz woanders hin abgeschweift. Für den Fall, dass sie sich nochmals Gedanken zum vorhergehenden Satz machte, sprechen wir von einer sogenannten Kontrollpause (vgl. auch Gyde Hanssen). Die Art der Pause, ob Orientierungs- oder Kontrollpause, ist für unseren Zweck hier jedoch nebensächlich. Tatsache ist, dass die Studentin nach dieser Pause zu schreiben begonnen hat: „Auch 
wenn es etwas banal klingen, mag, die sarkastische Aussage von Longanesi“, dann setzte sie drei Sternchen ${ }^{\star * *}$, ein Komma und setzte fort mit „Italien(... “. Mit den drei Sternchen kennzeichnete sie, dass sie hier für den italienischen Ausdruck „è innegabile che..." keine spontane Übersetzung parat hatte und auf diese Stelle später noch einmal zurückkommen wollte. Wir werden diese Art von Pause als „Wait-andsee“-Pause beschreiben und vielleicht anmerken, dass die Studentin in ihrem gesamten Prozess mehrere solcher „Wait-and-see“ Pausen eingelegt hat und diese Art von Pausen unseres Erachtens nach in Zukunft noch mehr erforscht werden sollten.

Sie schrieb weiter: „Italien beruht auf der Familie. Dies erklärt den schwachen“, korrigierte „den schwachen“ und ersetzte den Ausdruck mit „das schwache Bürgerbewusstsein “ und fährt fort: „ die Kunst sich ***“, korrigierte „die“ und ersetzte den Artikel durch „das chronische Misstrauen gegenüber dem Staat und den Ämtern welches schon seit Jahrhunderten empfinden." Als der Satz zu Ende ging, merkte die Studentin plötzlich, dass ihr noch ein Subjekt fehlte, sie kehrte im Satz zurück, plazierte den Curser nach dem Relativpronomen und fügte noch das Subjekt „die Italiener“ hinzu.

Nachdem sie sich den Satz ein weiteres Mal durchgelesen hatte (das ist wieder an der Pause ersichtlich), setzte sie den Curser in der Aufzählung zwischen „die Kunst sich ${ }^{* * *}$ " und „das chronische Misstrauen gegenüber (... “ an und fügte noch das Bindewort „und“ ein.

Kehren wir nochmals zu der Stelle zurück, an der es der Studentin gelungen ist, gleich drei kreative Übersetzungsvorschläge einzubauen. Dies passierte, wie das Abspielen der Logdatei und die Analyse ergeben haben, erst in der Revisionsphase. In der Revisionsphase nahm sie alle, wie im obigen Beispiel ersichtlichen, restlichen Änderungen vor und kam schliesslich auf die definitiven Lösungsvorschläge, die wir, gemäss unserer Definition und Auffassung von Kreativität im Übersetzungsprozess, als kreativ bezeichnen.

Bereits im Zuge dieser Ausführungen wird dem Leser vielleicht aufgefallen sein, dass es mitunter nicht leicht ist zu bestimmen in welcher Phase die kreative Lösung entstanden ist, weil - wie wir auch im vorhergehenden Beispiel sehen konnten - eine strenge Zuordnung meist nur schwer möglich ist.

Ein kreativer Übersetzungsvorschlag kann gänzlich in einer der beiden Phasen (Verlaufs- oder Revisionsphase) oder nur teilweise in der einen (Verlaufsphase) und teilweise in der anderen Phase (Revisionsphase) entstehen. Setzt sich ein definitiver kreativer Übersetzungsvorschlag aus zwei, oder mehreren Teillösungen zusammen, wird es problematisch mit Genauigkeit zu bestimmen, wann nun eigentlich der Zeitpunkt der Entstehung der kreativen Lösung gesetzt werden kann. In Anbetracht dieser Tatsache sind wir bei der Beschreibung der kreativen Momente peinlich genau vorgegangen und listen bei der Herkunft der einzelnen Übersetzungsvorschläge genau auf, ob es sich um eine Lösung handelt, die als solche zur Gänze in der einen oder anderen Phase oder nur teilweise in der einen und teilweise in der anderen Phase entstanden ist.

In Bezug auf die Datei der Studentin Tsch bedeutet dies, dass der erste kreative Lösungsvorschlag „auch wenn es etwas vereinfachend klingen mag“ [1] teilweise in der Verlaufsphase „auch wenn es etwas banal klingen mag“ und teilweise in der Revisionsphase entstanden ist, weil dort „banal“ mit „vereinfachend“ ersetzt wurde. Gleich verhält es sich mit dem zeiten Lösungsvorschlag „die Wahrheit der sarkastischen 
Aussage von Longanesi lässt sich nicht abstreiten“ [2], der zum Teil schon in der Verlaufsphase „die sarkastische Aussage von Longanesi ***“ entstanden ist und in zwei Versuchen in der Revisionsphase dann zu Ende geführt wurde: „lässt sich nicht abstreiten“ und etwas später „Wahrheit“. Der dritte Lösungsvorschlag ist als solcher wie er im Endeffekt als definitive Lösung stehenbleibt, erst in der Revisionsphase entstanden: „die Familie ist zentral in Italien“ [3]. In der Verlaufsphase hiess es noch: „Italien beruht auf der Familie“, was dann gänzlich korrigiert und die Endlösung ersetzt wurde. Der vierte Lösungsvorschlag „die Kunst sich durchzuschlängeln“ [4] ist dann wieder in beiden Phasen zustande gekommen: „die Kunst, sich ${ }^{\star \star \star ~ “ ~ i n ~ d e r ~}$ Verlaufsphase und das Verb „durchschlängeln“ in der Revisionsphase.

Nach der Analyse aller in unserem Korpus vorkommenden Übersetzungen ergab sich folgendes Bild:

\begin{tabular}{|c|c|c|c|c|}
\hline Name & Knr. & VP & RP & Bem. \\
\hline \multirow[t]{4}{*}{ TschI/D } & 1 & 88 & 88 & \\
\hline & 2 & $\mathscr{8}$ & 88 & \\
\hline & 3 & & 88 & Nach vollst. Korr. von L1. in VP \\
\hline & 4 & $\mathscr{8}$ & 88 & \\
\hline \multirow[t]{2}{*}{$\mathrm{Ki} \mathrm{I} / \mathrm{D}$} & 5 & & $\mathscr{8}$ & Nach 2 Korr. \\
\hline & 6 & $\mathscr{8}$ & 88 & \\
\hline $\mathrm{MeI} / \mathrm{D}$ & 7 & 88 & & Nach 2 Korr. \\
\hline Mo I/D & 8 & 88 & & L1 - L2 wieder zurück zu L1 \\
\hline $\mathrm{Pe} \mathrm{I} / \mathrm{D}$ & 9 & 88 & & 1. Lösung \\
\hline \multirow[t]{3}{*}{ Gi F/D } & 10 & 88 & & 1. Lösung \\
\hline & 11 & 88 & & 1. Lösung \\
\hline & 12 & 88 & & Nach 1 Korr. \\
\hline Kh F/D & 13 & $\mathscr{B}$ & & 1. Lösung \\
\hline \multirow[t]{2}{*}{$\mathrm{Me} F / \mathrm{D}$} & 14 & $\mathscr{8}$ & & \\
\hline & 15 & $\mathscr{8}$ & 88 & \\
\hline \multirow[t]{2}{*}{$\mathrm{Na} F / \mathrm{D}$} & 16 & 88 & & 1. Lösung \\
\hline & 17 & 88 & & Nach 2. Korr. \\
\hline Schweg F/D & 18 & $\mathscr{8}$ & $\mathscr{8}$ & \\
\hline Sp F/D & 19 & 88 & & 1. Lösung \\
\hline \multirow[t]{2}{*}{ Tsch F/D } & 20 & 88 & & Nach 3. Korr. \\
\hline & 21 & 88 & 28 & \\
\hline Schwen F/D & 22 & 88 & 88 & \\
\hline
\end{tabular}

Addiert man die Ergebnisse, so kommt man zum Schluss, dass 12 kreative Übersetzungsvorschläge in der Verlaufsphase, 8 sowohl in der Verlaufs- als auch Revisionsphase und nur 2 einzig in der Revisionsphase hervorgebracht wurden. In sieben von 22 Fällen war der kreative Übersetzungsvorschlag die erste hervorgebrachte Lösung, in den anderen Fällen kam dieser nach einer bis drei Korrekturen zustande. 
Graphisch lässt sich das zuletzt gesagte folgendermassen darstellen:

\section{Zum Entstehen der kreativen Lösungen - nach den einzelnen Phasen im Übersetzungsprozess}
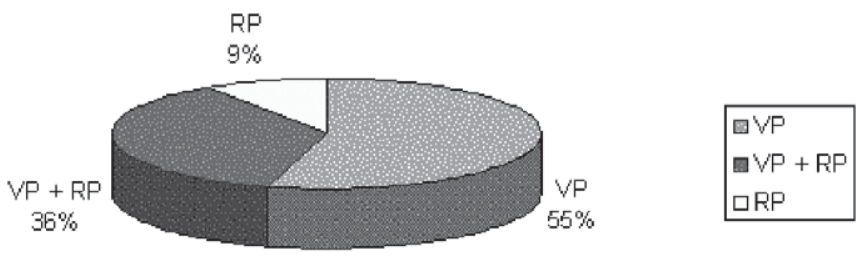

\section{Schlussfolgerungen}

Zusammenfassend kann somit postuliert werden, dass längere Revisionsphasen also ein starkes Indiz für kreative Übersetzungsleistungen sind, wobei lange Revisionsphasen als solche jedoch noch keine Garantie für Kreativität im Übersetzungsprozess darstellen. Was bringen uns diese Erkenntnisse? Wollten wir diese Resultate im Bereich der Übersetzungsdidaktik nützen, hiesse dies, dass Übersetzungsstudenten im Unterricht öfters dazu angehalten werden sollten, sich nach der eigentlichen Übersetzungsarbeit genügend (bzw. mehr) Zeit für Revisionen zu nehmen, da es eben gerade in dieser Phase des Übersetzungsprozesses zu kreativen (oder kreativeren) Übersetzungslösungen kommen kann.

Ausserdem ist schlussfolgernd festzuhalten, dass die meisten kreativen Lösungen aber erstmalig in der Verlaufsphase zu entstehen scheinen (55\%), ein grosser Anteil dann sowohl in der Verlaufs- als auch Revisionsphase (36\%) und nur ein kleiner Teil der Lösungen erst und einzig in der Revisionsphase (9\%). An dieser Stelle ist es uns nun auch möglich geworden, unsere letzte eingangs formulierte Hypothese zu prüfen, bei der es darum ging herauszufinden, ob der erste spontane Übersetzungsvorschlag, wie immer wieder vermutet, nicht bereits schon der kreativste sei. Und dies war, wie wir gesehen haben nur in knapp einem Drittel der Übersetzungsvorschlägen (sieben von 22) der Fall, weshalb diese Hypothese an diesem Stadium der Untersuchung wieder verworfen werden musste.

\section{NOTES}

1. Vgl. De Bono, Edward (1971): Laterales Denken. Ein Kursus zur Erschliessung Ihrer Kreativitätsreserven. Deutsch von Margaret Carroux und Wolfgang Eisermann. Reinbeck bei Hamburg: Rowohlt. Im Original: Lateral Thinking. A Textbook of Creativity. London: Ward Lock Educational 1970; De Bono,, Edward (1973): Beyond Yes and No. Harmondsworth: Penguin Books; Guilford, Joy Peter (1975); „Creativity: A Quarter Century of Progress“. In: Taylor, i. A. / Getzels, J. W. (Hrsg.): Perspectives in Creativity. Chicago: Aldine, 37-59. Hajek.

2. Was die Methoden zur Messung von Kreativität anbelangt, so kann man aufgrund der Arbeiten, in denen solche eingesetzt wurden, zwischen sprachlichen und sprachfreien Verfahren oder Skalen unterschieden. Zu den klassischen sprachlichen Verfahren zählen z. B. Mednicks RATs (Remote Associates Tests), bei denen jeweils drei vorgegebene Stimuluswörter mit geringer Assoziationsstärke untereinander mittels eines Wortes assoziativ verbunden werden, wobei kategoriale und klassifikatorische Lösungen nicht zugelassen sind und stets die Anzahl richtiger Wörter gemessen wird. Vgl. auch Mednick, S. A. \& Mednick, M. T. (1967): Examiner's manual: Remote Associations Tests. Boston: Houghton Mifflin; siehe. Auch Bowers, K. S.; Regehr, G. Balthazard, C. G. \& Parker, K. (1990): Intuition in the context of discovery. Cognitive Psychology, 22, 72-110; Weiters vielverwendet 
Guilfords DPTs (Divergent Production Tests) und UUTs (Unusual Uses Tests), der divergente Produktion als spezifisches Merkmal für Kreativität sieht, d. h. dass in diesen Tests nicht nur eine Lösung für ein gegebenes Problem gefunden werden soll, sondern möglichst viele Lösungsvorschläge und -alternativen. Unter den sprachfreien Verfahren zur Messung von Kreativität, davon abgeleitet (sie erfassen im Grunde die gleichen Merkmale, eben nur mit sprachfreiem Material) vor allem die TTCTs (Torrance Test of Creative Thinking) bekannt geworden.

3. Vgl. v. a. Kussmaul, Paul (2000): Kreatives Übersetzen, Tübingen: Stauffenburg Verlag Brigitte Narr Translation.

4. www.uns.umnw.ethz.ch/student/psych/ docs/Folien10_03_04.pdf. „Das kognitive Modell des Menschen 1: Denken und Problemlösen: Cogito ergo sum"

5. FUNKE Joachim (2003): “Kreatives Problemlösen: Konzept, Messung, Förderung”. Vortrag anlässlich der Graduiertentagung “Intelligenz und Kreativität”, veranstaltet vom Cusanuswerk, 26.30.11 .2003

6. Im Rahmen unserer Nachdiplomarbeit (unveröff.) zur Erlangung eines DEAs (diplôme d'études approfondies) en traduction; T. Heiden (Juni 2004): „ Wenn Translate aus dem Rahmen fallen Oder Kreatives Problemlösen beim Übersetze. Eine empirische Untersuchung ausgehend vom scenes-andframes Ansatz unter Anwendung von Translog 2000“. Ecole de Traduction et d'Interprétation, Université de Genève; sowie einer interdisziplinär angelegten FNRS Studie in Zusammenarbeit mit Prof. H. Lee-Jahnke (ETI, Université de Genève) und Dr. J.-M. Annoni und Dr. A. Khateb der Abteilung für Neurolinguistik am Kantons- und Universitätsspital Genf.

7. Guilford (1975) gilt, wie bereits angesprochen, unter Kreativitätsforschern allgemein als einer der beiden Urväter der Disziplin (neben Edward de Bono, 1971 und ist vor allem für seine vielzitierten und mittlerweile auch gängig gewordenen Begriffe des lateralen und divergenten Denkens bekannt geworden.

8. Nobelpreisträger für Wirtschaftswissenschaften, wird als einer der Begründer der künstlichen Intelligenz betrachtet. Er war es, der den Spezialisten der Kognitionswissenschaften mit dem von ihm entworfenen Computerprogramm General Problem Solver - ein Programm, das die kreativen Prozesse des menschlichen Denkens zu simulieren versucht - sozusagen den Grundstein für ihr Forschen legte. Der Titel eines seiner wichtigsten Werke, „The Sciences of the Artificial“ im Jahre 1969 veröffentlicht, liess bereits seine intellektuellen Ambitionen erahnen.

9. Vgl. Hussy (1992), Versuch inspiriert vom Schachtelproblem; Duncker (1935). Die elegantere Lösung, die Schachtel an die Tür zu pinnen und dann als Plattform für die Kerze zu verwenden, fanden nur wenige Personen. Das liegt laut Hussy an der Tatsache, dass ein Gegenstand funktional gebunden ist. Eine Schachtel wird gewohnheitsmässig als ein Behälter für andere Gegenstände aufgefasst, in dem Fall der Reissnägel. Es lag hier somit ein typisches Beispiel für eine Merkmalsfixierung vor. Der Bisoziationstheorie von Koestler zufolge wird die Lösungssuche zunächst mit Hilfe bewusster Prozesse angestrebt (rationale, logische aber auch unflexible Prozesse). Werden diese beendet, wird die Lösungssuche im Unbewussten fortgesetzt: Dabei erfolgt eine systematische Verknüpfung der Informationen bis gelegentlich die geeignete Lösung gefunden wird. Diese Neuverknüpfung nennt man Bisoziation (Assoziationen sind als alte Verknüpfungen zu betrachten).

10. Sie repräsentieren, nach Koestler, das eigentlich kreative Element aller Prozesse. Allerdings sind unbewusste (oder auch automatisierte, vgl. Hussy 2004) Prozesse Bestandteil eines jeden Problemlösungsvorgangs, wie z. B. ein Experiment von Eich (1984) zeigt.

11. Die Vpn sollte dabei die zentrale Szene von sechs Bildern mit jeweils einem Satz beschreiben: 12 B. „An einer Haltestelle stehen Leute, die sich in einen Bus drängen!“. Dabei war auf dem Bild für die Experimentalgruppe ein Geschäft mit dem Namen „Musikladen“ zu erkennen, für die Kontrollgruppe hingegen nicht.

12. Herzlicher Dank geht an dieser Stelle auch an Prof. Dr. Eddie Ronowicz von der Macquarie University Sydney.

13. vgl. dazu den Artikel „Selbstaufmerksamkeit im Übersetzungsprozess“, Hansen (2003).

14. Translog enthält, , zwei Funktionen: eine Grundfunktion „view facility“ sowie eine zweite Funktion, „replay facility“ genannt. Die „view facility“ zeigt eine Momentaufnahme der Log Datei, in dem Augenblick, in dem der Schreibprozess beendet ist, d. h. dass sie den gesamten Schreibprozess dokumentiert und so ein erstes Kontrollparameter bildet. Die „replay facility“ dient der dynamischen Wiedergabe des Schreibprozesses.

15. Vor der Übersetzung erhielten die Studenten jeweils kurze theoretische Erläuterungen zu Translog und prozessorientierten Forschungsarbeiten sowie eine praktische Einführung in das Programm, wobei der Versuchsleiter alle zu unternehmenden Schritte auf dem Computer vorführte und seine 
Präsentation auf einer Leinwand von allen Anwesenden mitverfolgt werden konnte. Um sich an den Umgang mit der Software zu gewö?hnen, nahmen die Studenten unter Anleitung des Versuchsleiters vor der Übersetzungsübung an einem Tipptest teil. Anschliessend konnten sie mit der Übersetzung beginnen, wobei ihnen keine Zeitlimite auferlegt wurde.

16. Dabei wurde ihnen verständlicherweise eine Zeitspanne vorgegeben, binnen derer sie die Übersetzung anzufertigen hatten.

17. Astrid Jensen (1999): „Time pressure in translation“, in: Probing the process in translation: methods and results. Copenhagen Studies in Language 24. Samfundslitteratur.

18. Bei der Bewertung des Übersetzungsprodukts wurde vom funktionalen Verständnis des Übersetzens im Hinblick auf die Anfertigung einer möglichst idiomatischen Übersetzung ausgegangen (Reiss, Hönig 1995, Nord 1998: Transparenz der Korrektur. In Snell-Hornby, M. / Hönig, H.G. / Kussmaul, P. / Schmitt, P.A. Hg. Handbuch Translation. Tübingen: Stauffenburg. 384-387).

19. Besonderes Augenmerk sollte dabei selbstverständlich den Translog Dateien der eben genannten Studenten geschenkt werden.

20. Auch sogenannte online-Revisionen wurden vorgenommen, d. h. während der Verlaufsphase selbst, wie im Logfile in der Folge ersichtlich war, und die Dauer dieser Phase nur die Revisionen nach Abschluss der Erstübersetzung beschreibt und nicht die Anzahl aller im Prozess vorkommenden Revisionen.

\section{BIBLIOGRAPHIE}

\section{ZUR TRANSLATION - KOGNITION}

Hönig, H. G. / Kussmaul, P. (1998a): „Einblicke in mentale Prozesse beim Übersetzen“, in M. Snell-Horby, H. G. Hönig, P. Kussmaul, P. A. Schmitt. (Hrsg.) (1998) Handbuch Translation, Tübingen: Stauffenburg Verlag. S. 170-178.

Kupsch-Losereit, S. (1998a): „Psycholinguistik“, in M. Snell-Horby, H. G. Hönig., P. Kussmaul, P. A. Sснмітт. (Hrsg.) (1998) Handbuch Translation, Tübingen: Stauffenburg Verlag, S. 64-66.

Kussmaul, P. (1995): Training the Translator. Amsterdam / Philadelphia, Benjamins.

LeE-JANHKe, H. (2001): „Aspects pédagogiques de l'évaluation en traduction“, in Meta, vol. 46-2, H. Lee-JanhKe (Hrsg.) S. 251-271, Montreal.

LEE-JANHKE, H. (1998): „L'introspection à haute voix: recherche appliquée“, in: Enseignement de la traduction et traduction dans l'enseignement. J. Delisle, H. LeE-Janhke (Hrsg.): Ottawa: Presses de l'Université, S. 155-184.

Rickeit, G. und H. Strohner (1993): Grundlagen der kognitiven Sprachverarbeitung: Modelle, Methoden, Ergebnisse, Tübingen, Francke Verlag.

Rickeit, G., Sichelschmidt L. und H. Strohner (2002): Psycholinguistik: Die Wissenschaft vom sprachlichen Verhalten und Erleben, Tübingen, Stauffenburg Verlag.

Risku, H. (1998a): Translatorische Kompetenz. Kognitive Grundlagen des Übersetzens als Expertentätigkeit. Studien zur Translation, Bd. 5, Snell-Hornby, Mary (Hrsg.). Tübingen: Stauffenburg Verlag

Risku, H. (1998b): „Kognitionswissenschaft“ M. Snell-Horby, H. G. Hönig., P. Kussmaul, P. A. Schмітт. (Hrsg.) (1998) Handbuch Translation, Tübingen: Stauffenburg Verlag, S. 119-122.

Schwarz, M. (1996): Einführung in die Kognitive Linguistik. 2, Auflage, UTB für Wissenschaft, Francke Verlag Tübingen und Basel.

Vega DE, M. (1984): Introducciòn a la Psicologia Cognitiva, Madrid, Alianza.

Wilss, W. (1988): Kognition und Übersetzen. Zur Theorie und Praxis der menschlichen und der maschinellen Übersetzung, Tübingen, Niemeyer.

\section{ZUR KREATIVITÄT}

Brodbeck, K.-H. (1999): Entscheidung zur Kreativität. Primus Verlag, Wissenschaftliche Buchgesellschaft, Darmstadt.

Craft, A., Jeffrey, B., and M. Leibling (2001): Creativity in Education, Continuum, British Library Cataloguing-in-Publication-Data, London. 
Damasio, A. (2001): "Some Notes on Brain, Imagination and Creativity", in K. H. Pfenninger, V.

R. Sнuвiк (Hrsg.) The Origins of Creativity. Oxford University Press, New York, S. 59-69.

De воno, E. (1971): Laterales Denken. Ein Kursus zur Erschliessung Ihrer Kreativitätsreserven, Deutsch von M. Carroux und W. Eisermann. Reinbek bei Hamburg, Rowohlt, Im Original Lateral Thinking. A Textbook of Creativity, London, Ward Lock Educational 1970.

De Bono, E. (1973): Beyond Yes and No. Harmondsworth, Penguin Books.

Gardner, H. (1982): Art, Mind and Brain: A Cognitive Approach to Creativity, New York, Basic Books.

Gardner, H., Csikszentminalyi, M., Feldman, H. (1994): Changing the World: A Framework for the Study of Creativity, Westport Conn., London, Praeger.

Gardner, H. (2001a): Les formes de la créativité: Einstein, Picasso, Gandhi, Übers. C. Larsonne, Paris: O. Jacob

Gardner, H. (2001b): “Creators: Multiple Intelligences", in K. H. Pfenninger, V. R. Shubik (Hrsg.) (2001), The Origins of Creativity, Oxford University Press, New York, S. 117-143.

Guilford, J. P. (1975): "Creativity: A Quarter Century of Progress", in I.A. TaYlor, J.W. GezTels (Hrsg.) Perspectives in Creativity, Chicago, Aldine, S. 37-59.

Lakoff, G. (1987): Women, Fire and Dangerous Things. What Categories Reveal about the Mind, Chicago, University of Chicago Press.

Osborn, A.F. (1963): Applied Imagination: Principles and Procedures of Creative Thinking (1963), New York, Scribner's rev. ed.

Preiser, S, (1976): Kreativitätsforschung, Darmstadt, Wissenschaftliche Buchgesellschaft.

Rouquette, M. L. (1997): La créativité, Paris, Presses universitaires de France, Que sais-je.

\section{ZUR KREATIVITÄT - TRANSLATION}

BALescu, I.; Stefanink, B.(2003): "Modèles explicatifs de la créativité en traduction" in META, 484,2003

Biser, E. (1993): „Kreatives Verstehen“, in J. Holz-Mänttari, C. Nord (Hrsg.): Traducere Navem. Festschrift für Katharina Reiss zum 70. Geburtstag. Tampere: Vertrieb durch Universitätsbibliothek Tampere (ohne Verlag), S. 219-227.

Heiden, T. (2004a): „Der Translator, ein kreativer Mittler zwischen konzeptueller Past-Fiction und Science-Fiction? Relevanz des Scenes-and-frames Ansatzes bei der Übersetzung ironisch-satirischer Texte." Beitrag an der vom 13. - 15. März 2003 an der Business School of Aarhus (Dänemark) abgehaltenen Eurokonferenz zum Thema „Knowledge Systems in Text and Translation“, In Hermes, Journal of Linguistics, no. 33-2004, S. 15-43.

Heiden, T. (2004b): „Wenn Translate aus dem Rahmen fallen. Oder Kreatives Problemlösen beim Übersetzen. Eine empirische Untersuchung ausgehend vom scenes-and-frames Ansatz unter Anwendung von Translog 2000“, Zur Erlangung eines DEA (Diplôme d'études approfondies) en traduction an der École de Traduction et d'Interprétation, Université de Genève, Unveröffentlichte Zusatzdiplomarbeit.

Krings, H. P. (1991): „Creativity in the Translation Process: Empirical Approaches“, in K. van Leuven-Zwart, Ton NaAukens (Hrsg.) Translation Studies: The State of the Art. Amsterdam/Atlanta: Rodopi. S. 91-101

Kussmaul, P. (1993): „Empirische Grundlagen einer Übersetzungsdidaktik Kreativität im Übersetzungsprozess“ in: Justa Holz-MänTtäri und Christiane Nord (Hrsg.). Traducere navem. Festschrift für Katharina Reiss zum 70. Geburtstag. Tampere: Universitätsbibliothek, S. $275-286$

Kussmaul, P. (1997): „Die Rolle der Psycholinguistik und der Kreativitätsforschung bei der Untersuchung des Übersetzungsprozesses" in: Fleischmann, Eberhard / Kutz, Wladimir / Schmitt, Peter A. (Hrsg.): Translationsdidaktik. Grundfragen der Übersetzungswissenschaft. Tübingen: Narr, S. 605-611

Kussmaul, P. (1999): „Die Übersetzung als kreatives Produkt“ in A. Gil, J. Haller, E. Steiner, H. Gerzymisch-Arbogast (Hrsg.): Modelle der Translation. Grundlagen für Methodik, Bewertung, Computermodellierung, Frankfurt am Main, Peter Lang, S. 177-189. 
Kussmaul, P. (2000a): „Types of Creative Translating“, in A. Chesterman, N. Gallardo, Y. Gambier (Hrsg.): Translation in Context. Selected Contributions from the EST Congress, Granada 1998, Amsterdam / Philadelphia: Benjamins S. 117-126.

Kussmaul, P. (2000b): Kreatives Übersetzen. Studien zur Translation. Tübingen: Stauffenburg

Markus, V. U. (2000): "Verstehen und Kreativität beim Übersetzen. Englische and deutsche Muttersprachler im Vergleich." In: Lebende Sprachen 1/2000, S. 1-8.

Riccardi, A. (1998): “Interpreting Strategies and Creativity", in A. Beylard-Ozeroff, J. Kralowa, Moser-Mercer, B. (Hrsg.): Translators Strategies and Creativity, Amsterdam / Philadelphia, John Benjamins.

\section{ZUM ÜBERSETZUNGSPROZESS}

Hansen, G. (1999): Probing the Process in Translation: Methods and Results, Copenhagen studies in language 24, Copenhagen, Samfundslitteratur.

Hansen, G. (2002): Empirical Translation Studies: Process and Product, Copenhagen studies in language 27, Copenhagen, Handelshojskolens Forlag.

Hansen, G. (2003): „Selbstaufmerksamkeit im Übersetzungsprozess“, Abstract des Beitrags an der vom 13. - 15. März 2003 an der Business School of Aarhus (Dänemark) abgehaltenen Eurokonferenz zum Thema „Knowledge Systems in Text and Translation“.

HönIG, H. G. (1991): "Holmes' Mapping Theory and the Landscape of Mental Translation Processes", in K. Van Leuven-Zwart et T. Naaijens (Hrsg.), Translation Studies: The State of the Art, Proceedings of the First James S. Holmes Symposium on Translation Studies, Amsterdam, Rodopi, S. 91-101.

JäÄskeläInen, R. (1987): What Happens in a Translation Proms: Think Aloud Protocols of Translation, Savonlinna, University of Joensuu, Savonlinna School of Translation Studies, Unveröffentlichte Dissertation.

JäÄSKelÄInEN, R. (1989a): “The Role of Reference Material in Professional vs. Non-professional Translation: A Think-Aloud Protocol Study", in S. Tirkkonen-Condit und Stephen Condit (Hrsg.), Empirical Studies in Translation and Linguistics, Joensuu, University of Joensuu, S. $175-200$.

JäÄskeläInen, R. (1989b): “Translation Assignment in Professional vs. Non-professional Translation: A Think-Aloud Protocol Study", in: Candace SÉGuinot (Hrsg.), The Translation Process, Toronto, H.G. Publications, S. 87-98.

JäÄskeläInEn, R. (1990): "Features of Successful Translation Processes A Think-Aloud Protocol Study”, Savonlinna, Savonlinna School of Translation Studies, University of Joensuu. Unveröffentlichte Diplomarbeit.

JäÄskelÄInEN, R. (1996), "Hard Work Will Bear Beautiful Fruit. A Comparison of two ThinkAloud Protocol Studies", Meta, Königs, Frank (Hrsg.) numéro special sur «Le(s) processus de la traduction", vol. 41, no 1 , S. 60-74.

JäÄskeläInen, R. (2002): „Think Aloud Protocol Studies into Translation. An Annotated Bibliography" in Target 14: 1, S. 107-136.

KöNigs, F. G. (1991): “Dem Übersetzen den Prozess machen? Psycholinguistische (Überlegungen zum Übersetzen und ihre didaktischen Konsequenzen", in E. IwASAKI (Hrsg.), Begegnung mit dem Fremden. Grenzen - Traditionen - Vergleiche, Akten des VIII. Internationalen Germanisten-Kongresses Tokyo 1990, Band 5, Munich, Iudicium Verlag, S. 132-141.

Krings, H. P. (1986): Was in den Köpfen von Übersetzern vorgeht: Eine empirische Untersuchung zur Struktur des Obersetzungsprozesses an fortgeschrittenen Französischlernern. Tübingen, Narr.

Kussmaul, P. und S. Tirkkonen-Condit (1995): “Think-Aloud Protocol Analysis in Translation Studies", in TER, vol. 8, $\mathrm{n}^{\circ}$ 1, S. 177-199.

LÖrscher, W. (1996): "A Psycholinguistic Analysis of Translation Processes" in META, Königs, Frank (Hrsg.) vol. 41, 1996. 
SÉguinot, C. (1989a): “The Translation Process: An Experimental Study", in C. SÉguinot (Hrsg.), The Translation Process, Toronto, H.G. Publications, School of Translation, York University, S. 21-53.

SÉGuinot, C. (1989b): "Understanding why Translators Make Mistakes", in TTR, vol. 2, nº 2. S. 73-81. 